\title{
Regulation of neural differentiation and synaptogenic factors by silencing of ubiquitin ligase Dorfin
}

\author{
Koichi Kawada $^{1 *}$, Ryo Saito ${ }^{2}$, Seisuke Mimori ${ }^{3}$, Yasunobu Okuma ${ }^{1}$ and Yasuyuki Nomura ${ }^{4}$ \\ ${ }^{1}$ Department of Pharmacology, Faculty of Pharmaceutical Sciences, Chiba Institute of Science, Choshi, Chiba 288-0025, Japan \\ ${ }^{2}$ Laboratory of Pharmacoinformitcs, School of Pharmacy and Graduate School of Ritsumeikan University, Kusatsu, Shiga 525-8577, Japan \\ ${ }^{3}$ Department of Clinical Medicine, Faculty of Pharmaceutical Sciences, Chiba Institute of Science, Choshi, Chiba 288-0025, Japan \\ ${ }^{4}$ Department of Pharmacology, Kurume University School of Medicine, Kurume, Fukuoka 830-0011, Japan
}

\begin{abstract}
Dorfin is an E3 ubiquitin ligase containing really $\mathrm{n}$ Gene-finger motif with a similar N-turminal region structure to the E3 ubiquitin ligase 3-hydroxyl-3methylglutaryl-coenzyme A reductase degradation 1 (HRD1). HRD1 is known to be related to the pathogenesis of neurodegenerative diseases. Dorfin is related to the pathogenesis of amyotrophic lateral sclerosis, but its involvement with other central nervous system diseases is not clear. In the present study, we investigated the effects of Dorfin on neuronal differentiation and synaptogenesis. The results showed that silencing Dorfin increased Hes1 and Pax6 mRNA levels and suppressed neuronal and glial differentiation, suggesting that the function of Dorfin's in differentiation may be similar to that of HRD1. In synaptogenesis, Dorfin silencing suppressed the expression of cell adhesion molecule 1 (CADM1), neurexin-1, and neuroligin-3, indicating that Dorfin normally upregulates synaptogenic factors, unlike HRD1. The difference between Dorfin and HRD1, in the case, might be due to their specific targets. It is possible that Dorfin targets ligands that have a PDZ domain such as CADM1, neurexin-1, and neuroligin-3. Overall, current results indicate that Dorfin promotes synaptogenesis via targeting to the ligands containing a PDZ domain.
\end{abstract}

\section{Introduction}

E3 ubiquitin-protein ligases ubiquitinate proteins, and has key roles for inducing the degradation of the unfolded proteins [1]. Ubiquitinated proteins also regulate neuronal development and function, and also interested in the pathogenesis of numerous neuronal diseases [2-4], such as amyotrophic lateral sclerosis and Parkinson's disease. We have previously reported that ubiquitin ligase, 3-hydroxyl3-methylglutaryl-coenzyme A reductase degradation 1 (HRD1) may regulate the neuronal differentiation and maturation during neuronal development [5]. HRD1 is a multiple transmembrane protein containing a Really Interesting New Gene (RING)-H2 finger domain and polyubiquitinates substrate proteins in endoplasmic reticulum (ER)-associated protein degradation system. HRD1 exists on the ER and suppresses apoptosis induced by ER stress [6]. Substrate proteins of HRD1 include 3-hydroxyl-3-methylglutaryl-coenzyme A reductase, T-cell receptor- $\alpha$, nuclear respiratory factor 1, amyloid precursor protein, Parkin-associated endothelin receptor-like receptor, and Huntingtin protein. Therefore, the functional impairment of HRD1 may be responsible for the idiopathic neuronal degenerative diseases. On the other hand, ring-finger protein $19 \mathrm{~b}$ was also identified as an ubiquitin ligase with a transmembrane region and RING finger domain, which is localized in the ER and is induced by ER stress, similar to HRD1. The ubiquitin ligase, double ring finger protein (Dorfin) is another E3 ubiquitin ligase similar to ring finger protein $19 \mathrm{~b}$ in N-terminal region structure. Dorfin (also known as ring finger protein 19a) has a RINGin between RING-fingers domain and can bind to mutated $\mathrm{Cu} / \mathrm{Zn}$ superoxide dismutase 1 and postsynaptic density-95 (PSD-95). Dorfin specifically degrades mutated superoxide dismutase 1 and inhibits neuronal cell death [7]. It is also able to interact with diverse substrates by forming a complex with the excitatory postsynaptic scaffolding protein PSD-95 [8]. Thus, Dorfin, like HRD1, is profoundly related with neuronal diseases. However, it is unknown whether Dorfin has an effect on neuronal differentiation and synaptogenesis. In the present study, we evaluated the effects of Dorfin on neuronal differentiation and synaptogenesis during neuronal development.

\section{Materials and methods}

\section{Antibodies and chemicals}

Rabbit polyclonal antibodies against cleaved Notch1 (Val1744) and $\mathrm{SH} 3$ and multiple ankyrin repeat domains 3 protein (Shank3)

Correspondence to: Koichi Kawada. Department of Pharmacology, Faculty of Pharmaceutical Sciences, Chiba Institute of Science, Choshi, Chiba 288-0025, Japan, Tel: +81-479-30-4674; E-mail: kkawada@cis.ac.jp

Key words: Dorfin, neuronal differentiation, synaptogenesis, ubiquitin ligase

Special Issue: Academic seeds for drugs

Katsura Takano

Associate Professor

Division of Veterinary Science

Graduate School of Life and Environmental Sciences

Osaka Prefecture University

Japan

Yasu-Taka Azuma, Ph.C., Ph.D.

Associate Professor

Principal Investigator at Laboratory on Veterinary Pharmacology Osaka Prefecture University

Japan

Received: June 12, 2017; Accepted: July 06, 2017; Published: July 10, 2017 
were purchased from Cell Signaling Technology, Inc. (Danvers, MA, USA) and Novus Biologicals (Littleton, CO, USA), respectively. Mouse monoclonal antibodies against nestin and neuronal nuclei (NeuN) and rabbit polyclonal antibodies against cell adhesion molecule 1 (CADM1), neurexin-1 (NRXN1), and neuroligin-3 (NLGN3) were purchased from Millipore Corp. (Temecula, CA, USA). A goat polyclonal antibody against Notch3 (M-20), a mouse monoclonal antibody against glyceraldehyde-3-phosphate dehydrogenase (GAPDH), and a rabbit polyclonal antibody against glial fibrillary acid protein (GFAP) were purchased from Santa Cruz Biotechnology, Inc. (Santa Cruz, CA, USA). Horseradish peroxidase-conjugated anti-mouse and anti-rabbit immunoglobulin $G$ antibodies were purchased from GE Healthcare (Buckinghamshire, UK). Alexa Fluor ${ }^{\circ}$ 488-conjugated anti-mouse immunoglobulin $G$ and Alexa Fluor ${ }^{\circ}$ 546-conjugated anti-rabbit immunoglobulin $\mathrm{G}$ antibodies were purchased from Life Technologies Corp. (Grand Island, NY, USA). Western Lightning Chemiluminescence Reagent Plus was obtained from Perkin Elmer Life Science Products, Inc. (Boston, MA, USA).

\section{Cell culture}

Culturing of mouse embryonal carcinoma P19 cells was performed as described previously [5]. Culture conditions and time schedules are shown in Figure 1. Briefly, the cells were maintained in Minimal Essential Medium- $\alpha$ (Life Technologies Corp.) containing 10\% (v/v) fetal calf serum (Life Technologies Corp.) until initial differentiation. These cells were then seeded at a density of $1 \times 105$ cells $/ \mathrm{mL}$ onto dishes coated with $0.2 \%$ agarose gel and cultured in Minimal Essential Medium- $\alpha$ containing $5 \%$ fetal calf serum and $0.5 \mathrm{mM}$ all-trans retinoic acid (Sigma-Aldrich Co., St. Louis, MO, USA) for $4 \mathrm{~d}$ under condition of floating cells. The cultured cells were seeded at a density of $2 \times 105$ cells $/ \mathrm{mL}$ in dishes coated with $75 \mu \mathrm{g} / \mathrm{mL}$ poly-L-lysine and cultured in Minimal Essential Medium- $\alpha$ containing 10\% fetal calf serum for $4 \mathrm{~d}$ under condition of adhering cells. All the cultures were maintained at $37^{\circ} \mathrm{C}$ in $95 \%(\mathrm{v} / \mathrm{v})$ humidified air and $5 \%(\mathrm{v} / \mathrm{v}) \mathrm{CO}_{2}$.

\section{Immunoblotting}

Immunoblot analysis was performed by using primary antibodies against Notch1 (1:1000), Notch3 (1:1000), nestin (1:1000), NeuN (1:2000), GFAP (1:2000), CADM1 (1:1000), Shank3 (1:1000), neurexin-1 (1:1000), neuroligin-3 (1:1000), and GAPDH (1:2000). Antibody-reactive proteins were detected by Western Lightning Chemiluminescence Reagent Plus and then quantified using an LAS3000 luminescent image analyzer (Fujifilm, Japan).

\section{Semiquantitative and real-time reverse transcriptase- polymerase chain reaction (RT-PCR)}

Total RNA was isolated from the differentiated P19 cells with TRI Reagent (Life Technologies Corp.) according to the manufacturer's instructions. cDNA was obtained by reverse transcription from the total RNA using a SuperScript VILO cDNA Synthesis Kit (Life Technologies Corp.). An aliquot of cDNA was then amplified by RT-PCR using GoTaq Hot Start Green Master Mix (Promega Corp., Madison, WI, USA). The nucleotide sequence of the primers is as described previously [5]. These experiments were performed with the same amount of cDNA.

mRNA expression was measured with a TaqMan-based real-time PCR assay using a 7500 Real-Time PCR System (Life Technologies Corp.).

\section{Data analysis}

All data were expressed as the mean \pm standard error of the mean, and statistical significance was determined by t-tests or Bonferroni or Dunn tests.

\section{Results}

\section{Efficiency of Dorfin knockdown}

Dorfin knockdown was carried out using small interfering RNA (siRNA) according to Figure 1 to evaluate whether Dorfin has an effect on the differentiation and synaptogenesis; real-time RT-PCR was performed to investigate the efficiency of Dorfin knockdown. The level of Dorfin mRNA was inhibited to only approximately $30 \%$ expression in Dorfin-suppressed cells at days 2 and 4 (Figure 2).

\section{Effects of Dorfin knockdown on the proneural factors}

To investigate whether the Dorfin is associated with the expression of proneural genes in P19 cells, a semiquantitative RT-PCR assay was designed to measure the expression of proneural genes, such as Hes1 and Pax6. Hes1 can maintain the stemness of neural stem cells that express Pax6, but leads cells that are Pax6-negative to astrocyte differentiation fate [9]. In the present study, the levels of Hes1 and Pax6 mRNA significantly increased in Dorfin-suppressed cells on day 4 (Figure 3A).

Undifferentiated cells overexpressing Hes1 have been found to suppress the activation of Notch signaling, followed by delayed neuronal differentiation [10,11]. Conversely, activated Notch converts from a gene repressor to a gene activator and regulates transcription

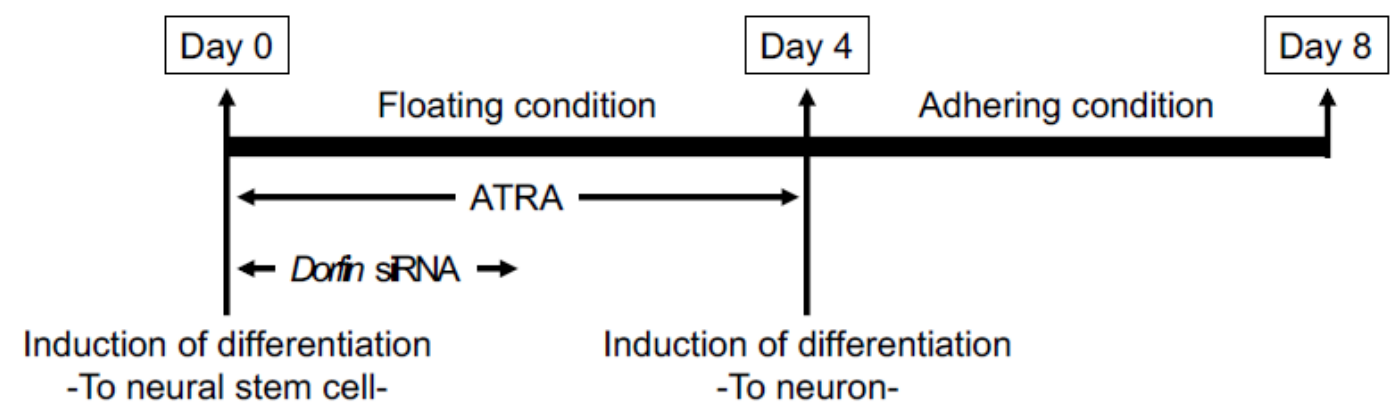

Figure 1. Culture of P19 cells. To induce the differentiation into neural stem cells, P19 cells were cultured with $0.5 \mu \mathrm{M}$ all-trans retinoic acid (ATRA) for $4 \mathrm{~d}$ under condition of floating cells. After, to induce the differentiation into neurons, these cells were cultured without ATRA for $4 \mathrm{~d}$ under condition of adhering. RNA interference was performed for $2 \mathrm{~d}$ with lipofection regents. siRNA, small interfering RNA. 


\section{Dorfin mRNA}
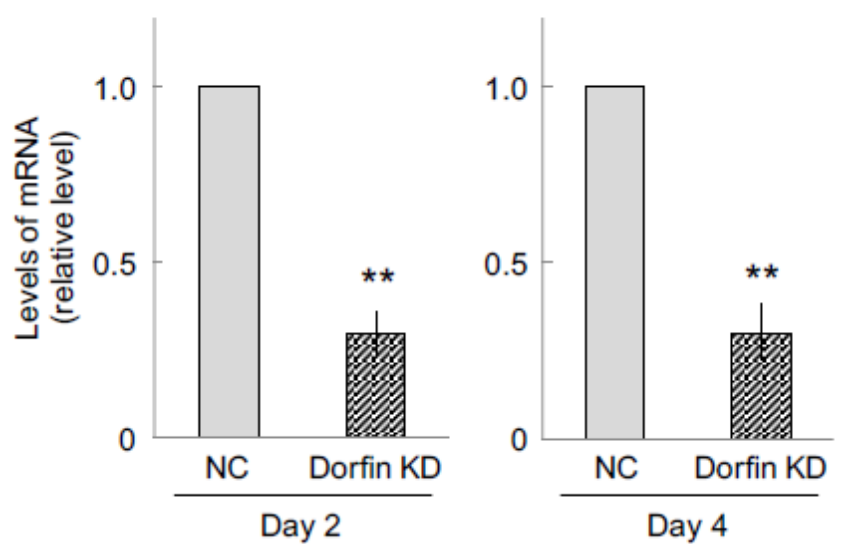

Figure 2. Efficiency of Dorfin knockdown (KD). Dorfin was transiently suppressed using Dorfin small interfering RNA, followed by neuronal differentiation using all-trans retinoic acid. Cells were harvested after 2 and $4 \mathrm{~d}$ and subjected to real-time RT-PCR to determine the efficiency of Dorfin knockdown at days 2 and 4 . Quantitative data are presented as levels relative to those for nontargeted controls $(\mathrm{NC})$. The values are presented as the mean \pm standard error of the mean from four independent experiments. $* * \mathrm{P}<0.01$, significantly different from $\mathrm{NC}$ values.

A

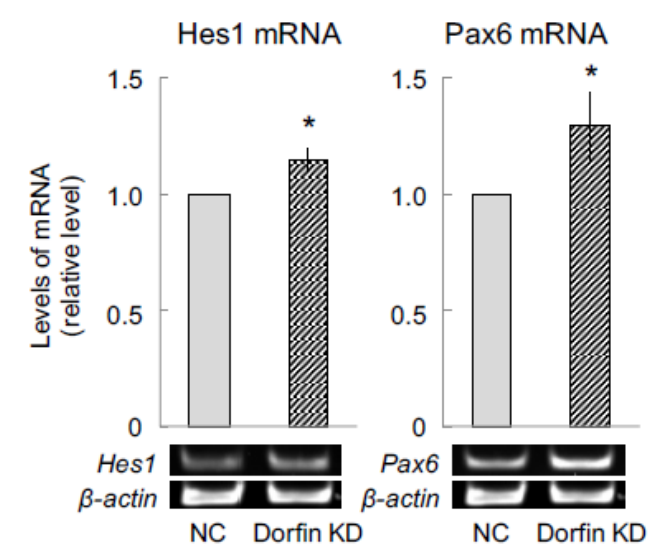

B

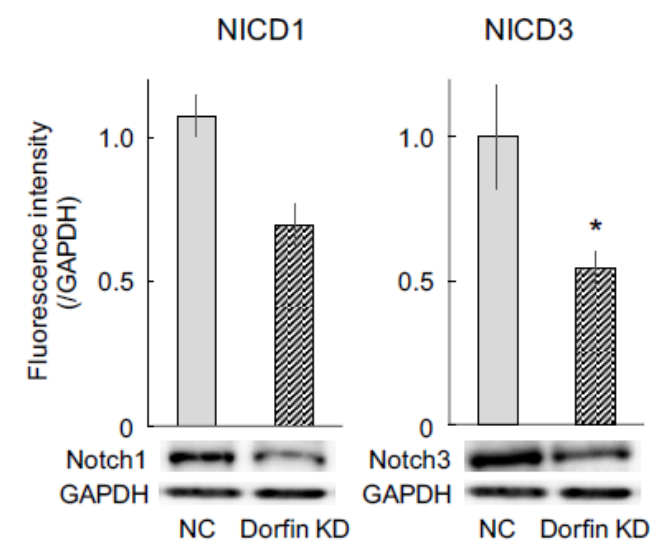

Figure 3. Expression of the factors related to neuronal differentiation. Dorfin was transiently suppressed using Dorfin small interfering RNA, followed by neuronal differentiation using all-trans retinoic acid. After $4 \mathrm{~d}$, cells were harvested, replated on dishes coated with poly-L-lysine, and incubated in the medium for $1 \mathrm{~h}$. Then, total RNA and lysates were retrieved from the cells and subjected to semiquantitative RT-PCR and immunoblotting, respectively, to determine signaling related with neuronal differentiation. Quantitative mRNA expression data are presented as levels relative to those for nontargeted controls (NC). $\beta$-Actin was used as an internal control. The values are presented as the mean \pm standard error of the mean from seven independent experiments. ${ }^{*} \mathrm{P}<0.05$, significantly different from NC values. NICD, Notch intracellular domain; KD, knockdown; GAPDH, glyceraldehyde-3-phosphate.

of Hes family genes [12]. To investigate whether Dorfin is associated with the activation of Notch signaling via proneural genes in P19 cells, immunoblotting was performed to measure the expression of activated Notch family proteins. The results showed that the levels of activated Notch1 (Notch intracellular domain 1, NICD1) and activated Notch3 (NICD3) proteins markedly decreased in Dorfin-suppressed cells on day 4 (Figure 3B).

\section{Effects of Dorfin knockdown on the neural differentiation}

To investigate whether Dorfin is involved in neuronal differentiation, immunoblotting was performed to measure the expression of neuronal marker proteins, such as nestin, NeuN, and GFAP. Before inducing neuronal differentiation, the expression of nestin was unchanged in Dorfin-suppressed cells on day 4 (Figure 4). However, after induction of differentiation, the expression of nestin tended to increase in Dorfin-suppressed cells on day 8 (Figure 4). In addition, the expression of neuron marker NeuN and glial marker
GFAP significantly decreased in Dorfin-suppressed cells on day 8 (Figure 4). Therefore, Dorfin-suppressed cells might maintain the state of undifferentiated cells.

\section{Effects of Dorfin knockdown on synaptogenic factors}

To investigate whether Dorfin is involved in synaptogenesis in neuronal cells, immunoblotting was performed to measure the expression of synaptogenic factors, such as CADM1, Shank3, NRXN1, and NLGN3. Current results showed that expression of CADM1 significantly decreased in Dorfin-suppressed cells on day 4 even though its expression was unchanged in Dorfin-suppressed cells on days 0 and 8 (Figure 5). However, the expression of Shank 3 remained unchanged at each time point (Figure 5). The expression of NRXN1 significantly decreased in Dorfin-suppressed cells on day 4 even though its expression was unchanged in Dorfin-suppressed cells on days 0 and 8 , similarly to CADM1 (Figure 5). The expression of CADM1 decreased with time in culture, while that of NRXN1 increased. On the 

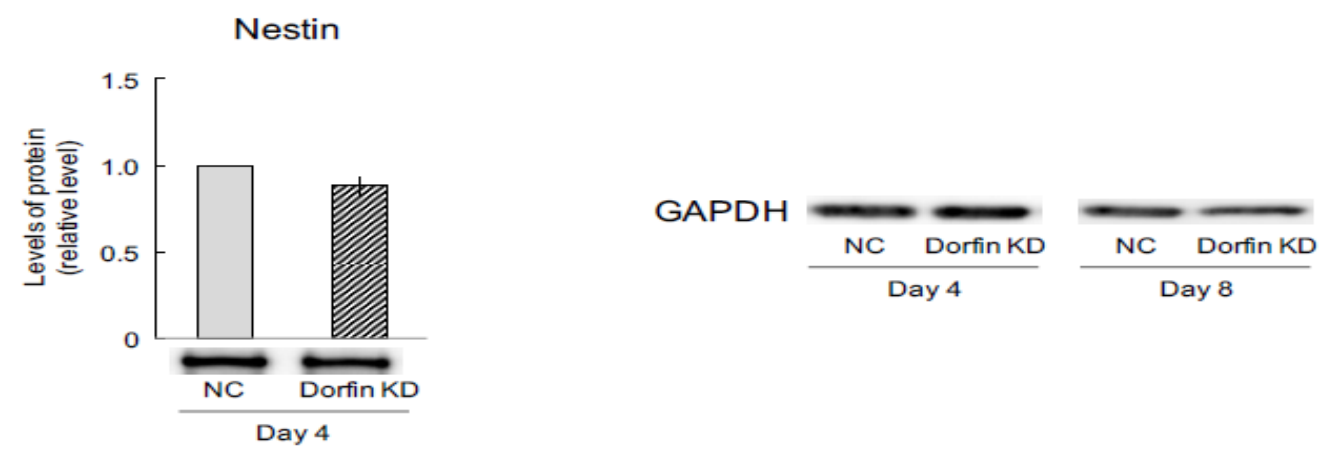

Nestin
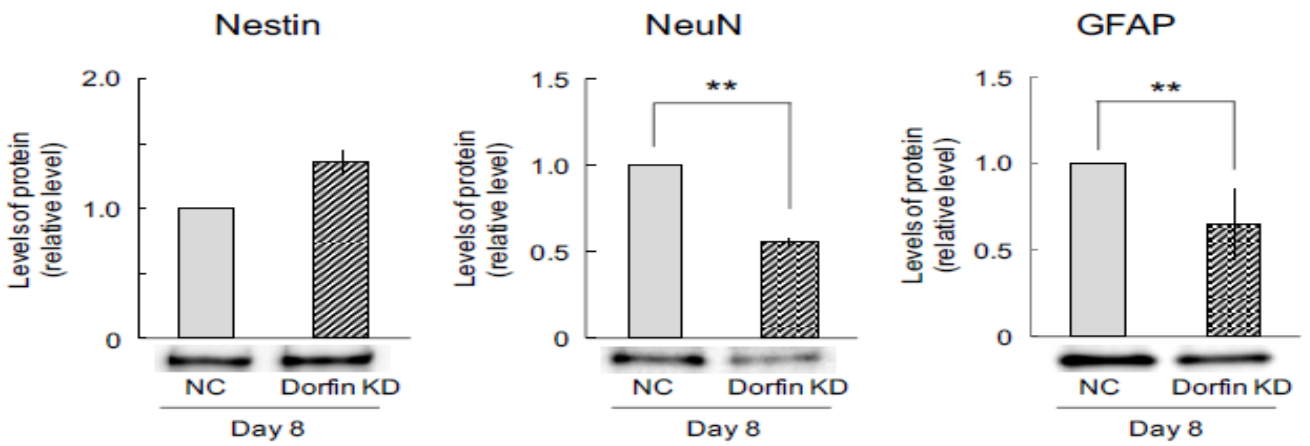

Figure 4. Number of neural cells in Dorfin-suppressed cells. Dorfin was transiently suppressed using Dorfin small interfering RNA, followed by neuronal differentiation using all-trans retinoic acid. After $4 \mathrm{~d}$, the cells were harvested, replated on dishes coated with poly-L-lysine, and incubated in the medium for $1 \mathrm{~h}$ (day 4 ) or cultured for an additional $4 \mathrm{~d}$ (day 8 ). Cell lysates were retrieved for immunoblotting of nestin, NeuN, and GFAP. The expression of internal control GAPDH has unchanged in any samples. Quantitative data are presented as levels relative to those for nontargeted controls $(\mathrm{NC})$. The values are presented as the mean \pm standard error of the mean from seven independent experiments. $* * \mathrm{P}<0.01$, significantly different from NC values. KD, knockdown.
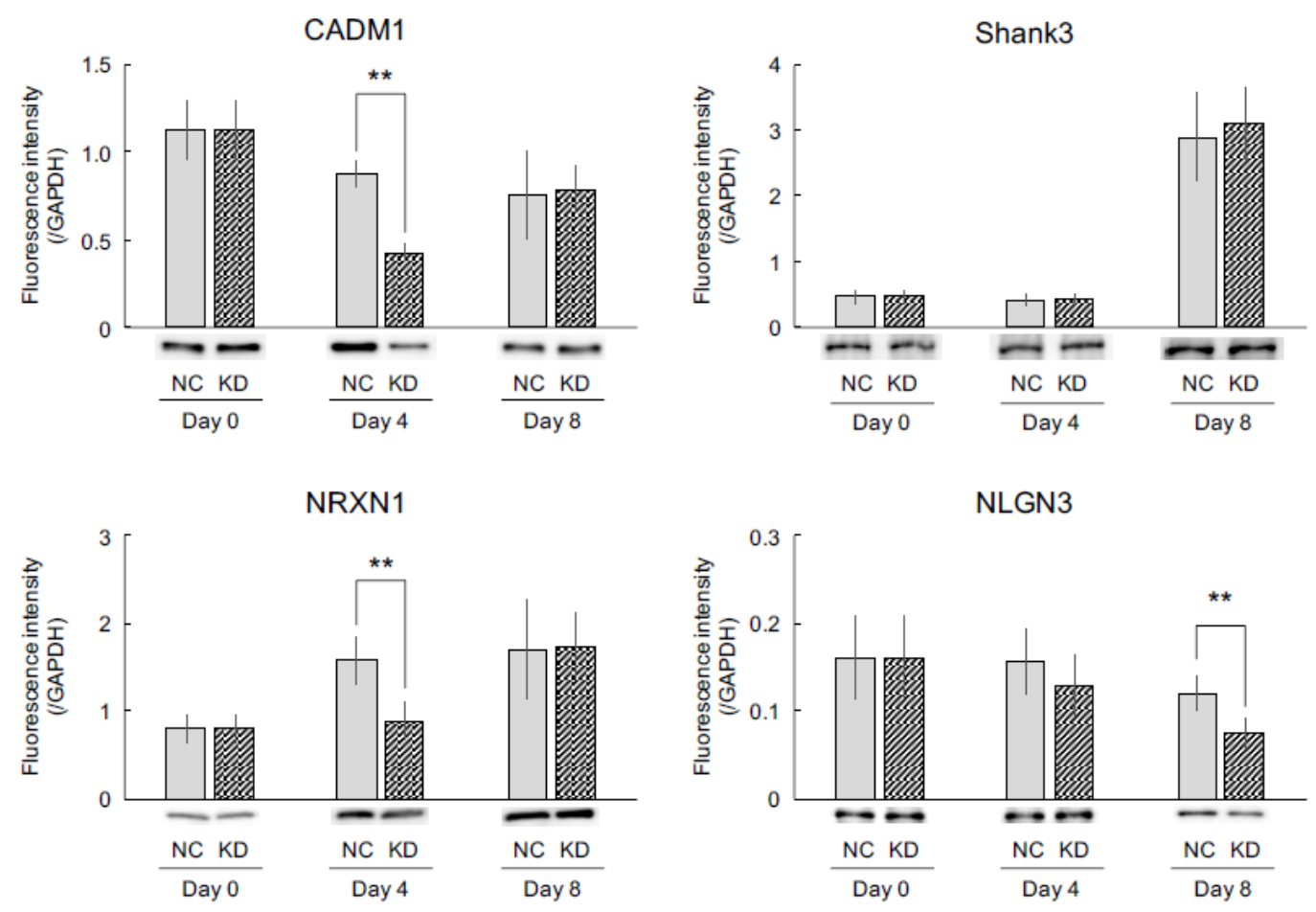

Figure 5. Expression of synaptogenic factors. Dorfin was transiently suppressed using Dorfin small interfering RNA, followed by neuronal differentiation using all-trans retinoic acid (day 0 ). After $4 \mathrm{~d}$, the cells were harvested, replated on dishes coated with poly-L-lysine, and incubated in the medium for $1 \mathrm{~h}$ (day 4 ) or cultured for additional $4 \mathrm{~d}$ (day 8 ). Cell lysates were retrieved for immunoblotting of CADM1, Shank3, NRXN1, and NLGN3. The expression of internal control GAPDH has unchanged in any samples. The values are presented as the mean \pm standard error of the mean from six independent experiments. $* * \mathrm{P}<0.01$, significantly different from nontargeted control (NC) values. KD, knockdown; GAPDH, glyceraldehyde-3phosphate. 
other hand, the expression of NLGN3 decreased in Dorfin-suppressed cells on day 8 even though its expression was unchanged in Dorfinsuppressed cells on days 0 and 4 (Figure 5).

\section{Discussion}

Dorfin is localized in ubiquitinated inclusions, such as Lewy bodies, in neurons and glial cells and plays a role in the quality control of intracellular proteins. Dorfin contains two RING fingers and one IBR domain at its $\mathrm{N}$-terminus. RING finger domain structures are coordinated with a $\mathrm{Zn}$ ion and function as ubiquitin-conjugating enzymes E2-binding sites. We previously found that the ubiquitin ligase HRD1 may cause abnormal neuronal differentiation and synaptogenesis during neural developmental process and was found to upregulate neuronal lineages by inducing the expression of proneural factors [5]. Additionally, increased HRD1 expression has been shown to upregulate neuronal and downregulate glial differentiation via ER stress [5]. In the present study, we were the first to investigate whether neuronal differentiation is regulated by the E3 ubiquitin ligase Dorfin, which is analogous to HRD1 in structure and function. Current results showed that Dorfin knockdown led to increases in the levels of Hes1 and Pax6 mRNA and decreases in the expression of NICD1 and NICD3 (Figure 3). Notch signaling is an important signal transduction pathway in developmental processes. Upon binding its ligand, Notch is separated into pieces, and its intracellular domain (NICD) translocates to the nucleus. The NICD plays an important role in the maintenance of the undifferentiated state, suppressing neuronal differentiation in neural stem cells. The expression of NICD1 and NICD3 is suppressed in Hes1-expressing undifferentiated cells [11]. While Pax6 expression maintains neural stem cells in an undifferentiated state via Hes1; its suppression led to induction of glial differentiation in neural stem cells through Hes1 [9]. In addition, Dorfin knockdown led to increased expression of nestin and decreased expression of NeuN and GFAP (Figure 4). Therefore, Dorfin might inhibit differentiation of neuronal lineages via Hes1 expression and Notch signaling, and suppress neuronal and glial differentiation by maintaining neural stemness.

We previously reported that ER stress can lead to decreased levels of Hes 1 and Pax6 mRNA but has no effect on the expression of NICD1 via increased HRD1 expression [5]. Additionally, ER stress-induced enhancement of HRD1 expression also increased the number of neurons [5]. In short, HRD1 may upregulate neuronal differentiation through proneural factors, like Hes1 and Pax6. Taken together, current and previous results indicate that the function of Dorfin is similar to that of HRD1 with regard to neuronal differentiation. Conceivably, this commonality is due to the RING finger domain found in both Dorfin and HRD1. Furthermore, disrupted deltex 1, another E3 ubiquitin ligase containing a RING finger domain, has been shown to reduce neuronal and glial differentiation [13], and RING finger-containing ubiquitin ligase Znf179 leads to cell cycle arrest via expression of p27, a cell cycle inhibitor [14]. Therefore, ubiquitin ligases containing RING finger motifs, such as Dorfin, may regulate neuronal and glial differentiation via expression of proneural factors and Notch signaling.

A number of synaptogenic factors are involved in the pathogenesis of autism spectrum disorders (ASD). CADM1, a member of the Ig superfamily, localizes to both sides of the synaptic cleft and promotes formation of presynaptic terminals and induction of the functional synapse. Shank3, a synaptic scaffolding protein, localizes in the postsynaptic density of excitatory synapses for formation, maturation, and maintenance of synapses. NRXN1 forms a trans-synaptic cell adhesion complex with postsynaptic NLGN and functions as a synaptic recognition molecule. In our previous preliminary study, overexpression of HRD1 suppressed the expression of synaptogenic factors, such as CADM1, NRXN1, and NLGN3, after neuronal differentiation (data not shown). Conversely, the present study found that silencing Dorfin decreased the expression of CADM1 and NRXN1 before neuronal differentiation (day 4). On the other hand, the expression of CADM1 and NRXN1 was unchanged in Dorfinsilenced cells after neuronal differentiation (day 8), despite a decrease in NLGN3. With respect to the expression of synaptogenic factors, the result of silencing Dorfin differs from that of silencing HRD1. One possible reason is that Dorfin might be interacting with synaptogenic factors through their PDZ domains (PSD-95, Discs-large, Zonula occludens-1). Dorfin has been previously shown to interact with the PDZ domain of PSD-95 but not with synaptic scaffolding molecules and glutamate receptor interacting protein 2 , which do not contain PDZ motifs [8]. Proteins containing a PDZ domain include PSD-95, multi-PDZ domain protein-1, calcium/calmodulin-dependent serine protein kinase, and Shank. CADM1 contains a type II PDZ-binding motif and is associated with multi-PDZ domain protein-1 $[15,16]$, and NRXN1 and NLGN3 can bind to calcium/calmodulin-dependent serine protein kinase and the PDZ3 domain of PSD-95, respectively [17]. We think that Shank3 has unchanged in Dorfin-suppressed cells because Shank3 contains PDZ motif in the structure, but not the ligand of PDZ domain [17]. Therefore, Dorfin may upregulate the expression of ligands that can bind PDZ domains.

Defects in synaptogenic factors, especially CADM1, NRXN1, NLGN3, and Shank3, are connected with the pathogenesis of ASD. However, Dorfin has never been reported to be associated with ASD. On the other hand, unfolded CADM1 inhibits elongation of dendrites by mainly accumulating in the ER, subsequently inducing failure of synaptogenesis in neurons accompanied by upregulation of C/EBPhomologous protein [18]. The interplay between presynaptic NRXN and their postsynaptic ligands functionally regulates synaptogenesis [19]. Additionally, dimerized NLGN induces mechanical clustering of NRXN and coordinates assembly of functional synapses [20]. Thus, it is possible that Dorfin contributes to the pathogenesis of ASD through the regulation of functional synaptogenic factors such as CADM1, NRXN1, and NLGN3. Such knowledge about Dorfin will further promote research on the development of therapeutic drugs for ASD.

\section{Acknowledgments}

The authors would like to thank Enago (www.enago.jp) for the English language review.

\section{Funding information}

This work was supported by Grants-in-Aid for Science Research (KAKENHI) 24790085 and 15K18879 from the Ministry of Education, Culture, Sports, Science and Technology of Japan (http://www.jsps. go.jp/english/e-grants/). The funding bodies played no role in the study design, data collection and analysis, decision to publish, or preparation of the manuscript.

\section{Competing interest}

The authors declare that they have no competing interests.

\section{References}

1. Friedlander R, Jarosch E, Urban J, Volkwein C, Sommer T (2000) A regulatory link between ER-associated protein degradation and the unfolded-protein response. Nat Cell Biol 2: 379-384. [Crossref] 
2. DiAntonio A, Haghighi AP, Portman SL, Lee JD, Amaranto AM, et al. (2001) Ubiquitination-dependent mechanisms regulate synaptic growth and function. Nature 412: 449-452. [Crossref]

3. Nakazawa S, Oikawa D, Ishii R, Ayaki T, Takahashi H, et al. (2016) Linear ubiquitination is involved in the pathogenesis of optineurin-associated amyotrophic lateral sclerosis. Nat Commun 7: 12547. [Crossref]

4. Shimura H, Schlossmacher MG, Hattori N, Frosch MP, Trockenbacher A, et al (2001) Ubiquitination of a new form of alpha-synuclein by parkin from human brain: implications for Parkinson's disease. Science 293: 263-269. [Crossref]

5. Kawada K, Iekumo T, Saito R, Kaneko M, Mimori S, et al. (2014) Aberrant neuronal differentiation and inhibition of dendrite outgrowth resulting from endoplasmic reticulum stress. J Neurosci Res 92: 1122-1133. [Crossref]

6. Kaneko M, Ishiguro M, Niinuma Y, Uesugi M, Nomura Y (2002) Human HRD1 protects against ER stress-induced apoptosis through ER-associated degradation. FEBS Lett 532: 147-152. [Crossref]

7. Niwa J, Yamada S, Ishigaki S, Sone J, Takahashi M, et al. (2007) Disulfide bond mediates aggregation, toxicity, and ubiquitylation of familial amyotrophic lateral sclerosis-linked mutant SOD1. J Biol Chem 282: 28087-28095. [Crossref]

8. Park H, Yang J, Kim R, Li Y, Lee Y, et al. (2015) Mice lacking the PSD-95-interacting E3 ligase, Dorfin/Rnf19a, display reduced adult neurogenesis, enhanced long-term potentiation, and impaired contextual fear conditioning. Sci Rep 5: 16410. [Crossref]

9. Sugimori M, Nagao M, Bertrand N, Parras CM, Guillemot F, et al. (2007) Combinatorial actions of patterning and HLH transcription factors in the spatiotemporal control of neurogenesis and gliogenesis in the developing spinal cord. Development 134: 16171629. [Crossref]

10. Kobayashi T, Mizuno H, Imayoshi I, Furusawa C, Shirahige K, et al. (2009) The cyclic gene Hes1 contributes to diverse differentiation responses of embryonic stem cells. Genes Dev 23: 1870-1875. [Crossref]
11. Kobayashi T, Kageyama R (2010) Hes1 regulates embryonic stem cell differentiation by suppressing Notch signaling. Genes Cells 15: 689-698. [Crossref]

12. Radtke F, Fasnacht N, Macdonald HR (2010) Notch signaling in the immune system. Immunity 32: 14-27. [Crossref]

13. Cheng YC, Huang YC, Yeh TH, Shih HY, Lin CY, et al. (2015) Deltex1 is inhibited by the Notch-Hairy/E(Spl) signaling pathway and induces neuronal and glial differentiation. Neural Dev 10: 28. [Crossref]

14. Pao PC, Huang NK, Liu YW, Yeh SH, Lin ST, et al. (2011) A novel RING finger protein, Znf179, modulates cell cycle exit and neuronal differentiation of P19 embryonal carcinoma cells. Cell Death Differ 18: 1791-1804. [Crossref]

15. Masuda M, Maruyama T, Ohta T, Ito A, Hayashi T, et al. (2010) CADM1 interacts with Tiam 1 and promotes invasive phenotype of human T-cell leukemia virus type I-transformed cells and adult T-cell leukemia cells. J Biol Chem 285: 15511-15522. [Crossref]

16. Fujita E, Tanabe Y, Imhof BA, Momoi MY, Momoi T (2012) A complex of synaptic adhesion molecule CADM1, a molecule related to autism spectrum disorder, with MUPP1 in the cerebellum. J Neurochem 123: 886-894. [Crossref]

17. Hung AY, Sheng M (2002) PDZ domains: structural modules for protein complex assembly. J Biol Chem 277: 5699-5702. [Crossref]

18. Fujita E, Dai H, Tanabe Y, Zhiling Y, Yamagata T, et al. (2010) Autsm spectrum disorder is related to endoplasmic reticulum stress induced by mutations in the synaptic cell adhesion molecule, CADM1. Cell Death Dis 1: e47. [Crossref]

19. Uemura T, Shiroshima T, Maeda A, Yasumura M, Shimada T, et al. (2017) In situ screening for postsynaptic cell adhesion molecules during synapse formation. $J$ Biochem. [Crossref]

20. Shipman SL, Nicoll RA (2012) Dimerization of postsynaptic neuroligin drives synaptic assembly via transsynaptic clustering of neurexin. Proc Natl Acad Sci U S 109 19432-19437. [Crossref]

Copyright: (C2017 Kawada K. This is an open-access article distributed under the terms of the Creative Commons Attribution License, which permits unrestricted use, distribution, and reproduction in any medium, provided the original author and source are credited. 